\title{
Tumor de Frantz: Relato de um Caso
}

\author{
Frantz's Tumor: Report of a case
}

\author{
Thiago Andrade de Macedo ${ }^{1}$, Sabas Carlos Vieira ${ }^{2}$, Adriano Machado de Oliveira ${ }^{3}$, Eid Gonçalves Coelho ${ }^{4}$, Lina Gomes dos Santos ${ }^{5}$ e \\ Jerúsia Oliveira Ibiapina de Santana ${ }^{6}$
}

\section{Resumo}

O tumor de Frantz é uma neoplasia rara do pâncreas. Ocorre principalmente em pacientes jovens e apresenta um bom prognóstico. Pouco mais de 300 casos foram relatados na literatura mundial. Os autores apresentam um caso de Tumor de Frantz, em uma paciente do sexo feminino cuja apresentação clínica foi de massa abdominal palpável. A tomografia computadorizada (TC) associada à ultrassonografia (US) evidenciaram presença de massa sólidocística. Dois terços destes tumores ocorrem no corpo e cauda do pâncreas, e apesar do crescimento excessivo para fora dos limites pancreáticos, raramente invadem estruturas vasculares ou órgãos adjacentes, o que correspondeu aos achados intraoperatórios do caso. A paciente foi submetida a pancreatectomia corpo-caudal com preservação do baço. À microscopia foram evidenciadas cavidades císticas com ocasionais estruturas papilíferas. A paciente encontra-se sem evidência da doença 24 meses após a operação. Enfatiza-se a necessidade de se considerar o tumor de Frantz no diagnóstico diferencial de massa abdominal em pacientes jovens.

Palavras-chave: tumor de Frantz; neoplasias pancreáticas; diagnóstico diferencial; cirurgia; prognóstico.

\section{Abstract}

Frantz's tumor is a rare neoplasia of the pancreas that occur mainly in teenage patients with a good prognosis. A little over 300 cases have been reported in the literature worldwide. The autors present a case of Frantz's tumor on a female patient, whose presentation was palpable abdominal mass. CT scans and ultrasound imaging showed a solid cystic mass. Two thirds of these tumors occur at the body or tail of the pancreas, and in spite of the excessive growth around the pancreas, it rarely invades vascular structures or adjacent organs, what could have been seen intraoperatively in this case. The patient was submitted to a partial pancreatectomy of body and tail with preservation of the spleen. Microscopic examination displayed evident cystic cavities with occasional papillary structures. The patient is disease-free 24 months after the operation. We emphasize the need to consider tumor of Frantz as a differential diagnosis for abdominal masses in youngsters.

Key words: Frantz's tumor; pancreatic neoplasms; differential diagnosis; surgery; prognosis.

\footnotetext{
${ }^{1}$ Estudante de Medicina Universidade Federal do Piauí - UFPI

${ }^{2}$ Professor de Cirurgia da UPFI. Especialista em Cancerologia

${ }^{3}$ Residente em Cirurgia Geral- Hospital Getúlio Vargas -HGV

${ }^{4}$ Residente em Cirurgia Geral- Hospital Getúlio Vargas -HGV

5Patologista- SPCC Sociedade Piauiense de Combate ao Câncer- Hospital São Marcos

${ }^{6}$ Patologista- SPCC Sociedade Piauiense de Combate ao Câncer- Hospital São Marcos

Trabalho realizado no Departamento de Clínica Geral- Hospital Getúlio Vargas (HGV) / Clínica Cirúrgica (HGV) / Hospital São Marcos -

SPCC / Internato da Universidade Federal do Piaú (UFPI)

Endereço para correspondência: Dr. Sabas Carlos Vieira - Rua Seis, 2106, Casa V - Residencial Tropical Park - CEP: 64055150 - e-mail:

sabasmarcia@uol.com.br)
} 


\section{INTRODUÇÃO}

O tumor de Frantz é uma neoplasia sólido-cística do pâncreas que ocorre em mulheres jovens e que apresenta um bom prognóstico. Pouco mais de 300 casos foram relatados na literatura mundial ${ }^{1}$. O tumor apresenta etiopatogenia desconhecida ${ }^{2}$, baixo potencial de malignidade e bom prognóstico, mesmo quando os pacientes apresentam invasão local ou metástases. Manifesta-se clinicamente com massa abdominal de crescimento lento, com ou sem dor abdominal. O tratamento é cirúrgico com ressecção do tumor e/ou pancreatectomia. Recorrências locais e metástases podem ocorrer, podendo ser resgatadas com cirurgia el ou quimioterapia ${ }^{3}$.

Relatamos um caso de Tumor de Frantz submetido à ressecção cirúrgica. Enfatiza-se a raridade do caso e a necessidade de se considerar o tumor de Frantz no diagnóstico diferencial de massa abdominal em pacientes jovens.

\section{RELATO DO CASO}

Uma paciente do sexo feminino com 19 anos de idade foi admitida na Clínica Cirúrgica do Hospital Getúlio Vargas com queixa de dor progressiva no hipocôndrio esquerdo. Ao exame físico , apresentavase com bom estado geral, hipocorada $(+/ 4+)$, hidratada, anictérica e afebril. $\mathrm{O}$ exame cardiopulmonar foi normal. O exame do abdômen evidenciou a presença de abaulamento localizado em HE, com massa palpável, dolorosa, e espaço de Traube ocupado.

Os exames laboratoriais revelaram hematimetria e função renal normais, assim como a radiografia de tórax. A tomografia computadorizada (TC) associada à ultrassonografia ( US ) evidenciaram presença de massa sólido-cística com contornos irregulares, localizada no hipocôndrio esquerdo, com 13,3 x 10,6 cm de diâmetro, sugestiva de tumor sólido-cístico do pâncreas.

A paciente foi submetida a uma laparotomia, encontrando-se uma volumosa massa de consistência sólido-cística, de contornos bem delimitados, localizada no corpo do pâncreas, aderida à veia esplênica em toda sua extensão, sem evidência de invasão da mesma. Realizou-se uma pancreatectomia corpo-caudal com preservação esplênica. Os achados anatomopatológicos revelaram, à macroscopia, um tumor de $220 \mathrm{~g}$, medindo $11,0 \times 10,0 \times 8,0 \mathrm{~cm}$ com superfície externa rugosa e parda. À microscopia, proliferação de células redondas, regulares e monomórficas em arranjos frouxos, por vezes em disposição rosetóide. Cavidades císticas com ocasionais estruturas papilíferas. Presença de necrose e índice mitótico $<1 / 10$ CGA. Seis linfonodos examinados apresentaram processo inflamatório inespecífico.

A paciente evoluiu bem no pós-operatório, recebendo alta no sétimo dia. Encontra-se sem evidência da doença 24 meses após a operação.

\section{DISCUSSÃO}

O tumor de Frantz ocorre predominantemente em crianças e mulheres jovens, geralmente na segunda e terceira décadas de vida ${ }^{5}$. A apresentação clínica freqüentemente é de tumoração abdominal, com o paciente em bom estado geral. A presença de icterícia é infreqüente, mesmo nos tumores localizados na cabeça do pâncreas ${ }^{6}$. Microscopicamente, o corte transversal exibe amostras de áreas sólidas e papilares. $\mathrm{Na}$ área papilar, as células tumorais apresentam mitocôndrias abundantes, com ou sem grânulos neurossecretores ${ }^{6}$.

No caso relatado, a paciente era do sexo feminino, encontrava-se na segunda década de vida e apresentava como queixas principais dor e massa palpável em hipocôndrio esquerdo, semelhante ao relato na literatura. A ultrassonografia abdominal e a tomografia computadorizada são os exames mais utilizados para o diagnóstico. $\mathrm{Na}$ ultrassonografia abdominal a massa pancreática é geralmente heterogênea, com componentes císticos ${ }^{6}$, aspecto que depende do grau de hemorragia ou necrose presente. No diagnóstico pré-operatório, a radiografia simples de abdome mostrou presença de massa com calcificaçôes no abdome superior. A ultrassonografia e tomografia computadorizada do abdome evidenciaram massa pancreática encapsulada de consistência sólido-cística.

Dois terços destes tumores ocorrem no corpo e cauda do pâncreas, e apesar do crescimento excessivo para fora dos limites pancreáticos, raramente invadem estruturas vasculares ou órgãos adjacentes, o que correspondeu aos achados intraoperatórios do caso. O tipo de tumor é geralmente diagnosticado somente após a ressecção cirúrgica, sendo a maioria benigna ou com baixo grau de malignidade, mesmo com extensão para a veia porta ou o duodeno ${ }^{6}$.

O diagnóstico diferencial inclui outras lesões pancreáticas como: tumor isolado não funcional, carcinoma de células acinares, cistoadenoma papilífero, cistoadenoma seroso, carcinoma pancreático infantil e pseudocisto ${ }^{2,8}$. 
O comportamento evolutivo contrasta muito com a apresentação do adenocarcinoma pancreático que, no momento do diagnóstico, geralmente se apresenta localmente avançado ${ }^{7}$.

O tratamento recomendado, devido ao baixo grau de malignidade do tumor e excelente prognóstico, é conservador, com ressecção completa do tumor, haja vista a alta possibilidade de cura. A rádio-quimioterapia não é utilizada para o tratamento padrão ${ }^{1}$. Assim, ressecção cirúrgica é o tratamento definitivo do tumor de Frantz ${ }^{7}$.

As recidivas podem ocorrer, sendo necessário acompanhamento prolongado do paciente ${ }^{1}$. Metástases à distância para o fígado, pulmão ou pele ocorrem predominantemente em mulheres idosas e estão associadas a alta mortalidade ${ }^{6}$.

Observamos, neste caso, que tanto a apresentação clínica quanto a radiológica apontam para o diagnóstico de tumor de Frantz, o qual foi confirmado pela análise histopatológica. Em relação ao prognóstico, a presença de cápsula bem formada, com ausência de metástases e a ressecção completa da massa foram importantes para a evolução favorável.

\section{REFERÊNCIAS BIBLIOGRÁFICAS}

1. Berretta S, Barbagallo E, D'agata A, Berretta M. Frantz's solid cystic papillary pancreatic carcinoma. Minerva Chir
2001;56(4):413-9.

2. Snajdauf J, Pycha K, Rygl M, Kocmichova B, Kodet R, Koutecky J, et al. Papillary cictic and solid tumor of the pancreas surgical therapy with the use of CUSA, and a review of the pediatric literature. Eur J Pediatr Surg 1999;9(6):416-9.

3. Rebhandl W, Felberbauer FX, Puig S, Paya K, Hochschomer S, Barlan M, et al. Solid-pseudopapillary tumor of the pancreas (Frantz tumor) in children: report of four cases and review of the literature. J Surg Oncol 2001;76(4):289-96.

4. Machado MCC, Monteiro da Cunha JE, Bacchella T, Jukemura J, Penteado S, Zerbini MCN, et al. Tumor de pâncreas (neoplasia epitelial e cística do pâncreas): estudo de três casos. Rev Hosp Clín Fac Med São Paulo 1993;48(1):29-34.

5. Rivera M, Ortiz VN, Duran N, Trujillo O. Solid and papillary neoplasm of the pancreas: a case presentation. Bol Assoc Med PR1998;90(4/6):91-2.

6. Yang YJ, Chen JS, Chen CJ, Lin PW, Chang KC, Tzeng CC. Papillary cistic tumor of the pancreas in chindren. Scand J Gastroenterol 1996;31(12):1223-7.

7. Meniconi MT, Sousa MVA, Rodrigues ALS, Mancero JMP, Quireze C Jr, Cardoso ES, et al. Tumor de Frantz, relato de dois casos: medidas terapêuticas e prognóstico. Arq Gastroenterol 1997;34(1):43-8.

8. Torres FC, Hidalgo RR, Benitez NN, Rodriguez SJ, Llanos VL, Muñoz C, et al. Tumor de Frantz neoplasia de un caso y revisión de la literatura. Rev Ecuat Cancerol 1996;3(1):45-9. 